\title{
Tormenta tiroidea: revisión de dos casos en Hospital de San José, Bogotá
}

\section{William Rojas ${ }^{1}$ Henry Tovar $^{2}$ Alejandra Rivera ${ }^{3}$}

${ }^{1}$ Médico Internista, Endocrinólogo, Profesor y Jefe de posgrado de Endocrinología de la Fundación Universitaria de Ciencias de la Salud.

${ }^{2}$ Médico Internista, Endocrinólogo, Profesor de Endocrinología Fundación Universitaria de Ciencias de la Salud.

${ }^{3}$ Residente de Medicina Interna, Fundación Universitaria de Ciencias de la Salud.

Fecha de recepción: 17/07/2015

Fecha de aceptación: 01/10/2015

\section{Introducción}

$\mathrm{L}$ a tormenta tiroidea es una exacerbación aguda de hipertiroidismo que se presenta por una repentina liberación de hormonas tiroideas hacia la circulación sistémica ${ }^{(1)}$. Es una afección potencialmente mortal que requiere tratamiento de emergencia. Se manifiesta por la descompensación de múltiples órganos, que a menudo se desencadena por estrés severo. Es una entidad poco común, con una incidencia reportada de 0,2 casos $/ 100.000$ habitantes y tasas de mortalidad alta de $10 \%$ a $30 \%{ }^{(2)}$. No obstante, el diagnóstico y el tratamiento oportunos pueden modificar ese desenlace al mejorar la supervivencia en los pacientes. Se ha descrito que la intervención temprana puede disminuir la mortalidad al $10 \%$ de los $\operatorname{casos}^{(3)}$. En ese orden de ideas, es importante dar a conocer dicha patología, su presentación, el enfoque diagnóstico y el manejo oportuno para modificar el desenlace de los pacientes en nuestras instituciones, es el motivo de la presentación de dos casos de tormenta tiroidea en el Hospital de San José en Bogotá.

Palabras clave: tormenta tiroidea, tormenta tirotóxica, hipertiroidismo.

\section{Caso 1}

Paciente de 39 años quien asiste a consulta externa de endocrinología por cuadro clínico de 18 meses de evolución caracterizado por insomnio, caída del cabello, cejas y pestañas, asociado a temblor distal, palpitaciones, diaforesis, pérdida de peso y episodios de diarrea frecuente, además irregularidades del ciclo menstrual y síntomas de depresión con ideación suicida. Presenta exacerbación de los síntomas hace 20 días con aumento de los episodios de palpitaciones. Tiene antecedente de hipertiroidismo hace dos años, manejada inicialmente con metimazol $25 \mathrm{mg}$ /día, propranolol $40 \mathrm{mg}$ cada 12 horas, pero fue cambiado por propiltiouracilo $500 \mathrm{mg} /$ día por exantema cutáneo. En el momento de la consulta se encontraba taquicárdica con frecuencia cardiaca 149 latidos por minuto, temperatura de $37,6^{\circ} \mathrm{C}$, a la inspección se evidencia aumento de tamaño de glándula tiroides, mucosas húmedas, presenta edema de miembros inferiores sin fóvea y al examen neurológico con taquilalia, taquipsiquia, nerviosismo y agitación. Se tomó hemograma, el cual estaba normal, transaminasas normales, TSH: 0,005 , T4 libre: 5,97 , por lo que se considera que cursa con tormenta tiroidea con escala de Burch y Wartofsky de 50 puntos (temperatura de $37,6^{\circ} \mathrm{C}$ : 5 puntos, agitación: 10 puntos, diarrea: 10 puntos, frecuencia cardiaca de 149 lpm: 25 puntos), motivo por el cual se decide hospitalizarla, se inicia manejo con propiltiouracilo $500 \mathrm{mg}$ dosis inicial y continuar a $200 \mathrm{mg}$ cada 4 horas por vía oral, propranolol 80 mg vía oral cada 12 horas y dexametasona $2 \mathrm{mg}$ intravenosos cada 6 horas. Paciente con adecuada evolución clínica, con disminución de la frecuencia cardiaca posterior a siete días de tratamiento, además con mejoría de síntomas neurológicos y normalización de la temperatura. Dada la adecuada respuesta clínica, se dio de alta a la paciente con orden para tratamiento con yodo radiactivo de manera ambulatoria.

\section{Caso 2}

Paciente de 22 años en estado de embarazo de 37 semanas con antecedente de enfermedad de Graves en manejo con metimazol $5 \mathrm{mg}$ cada 12 horas y propranolol 40 mg día, quien consulta por cuadro clínico de 15 días de evolución caracterizado por diaforesis, diarrea en múltiples ocasiones, nerviosismo, caída del pelo, palpitaciones y disnea. Familiares refieren que ella ha presentado letargia por lo cual consulta al servicio de urgencias de ginecología. Paciente que ingresa con taquicardia de 140 latidos por minuto, temperatura de $37,5^{\circ} \mathrm{C}$, con exoftalmos bilateral de predominio derecho, bocio grado III, piel húmeda y temblor distal, además de letargia. Se toman niveles de TSH: <0,015, T4L: 66,2, con escala de Burch y Wartofsky de 60 puntos (temperatura de $37,5^{\circ} \mathrm{C}: 5$ puntos, letargia: 20 puntos, diarrea: 10 puntos, frecuencia cardiaca $140 \mathrm{lpm}: 25$ puntos), por lo que se confirma diagnóstico de tormenta tiroidea. Se hospitaliza, se confirma bienestar fetal y dado el riesgo actual y el riesgo de efectos deletéreos hacia el feto se decide continuar medicación antitiroidea con metimazol en dosis 
de $1 \mathrm{mg} / \mathrm{kg} /$ día. Además se indicó inicio de dexametasona 8 mg intramuscular cada 8 horas durante 3 días para bloquear la conversión de T4 a T3 y mejorar los síntomas de la oftalmopatía, propranolol $40 \mathrm{mg}$ vía oral cada 12 horas. Paciente que durante la hospitalización no evidencia causa aparente de descompensación, tiene adecuada respuesta al tratamiento al cabo de ocho días, con mejoría de sintomatología dada por disminución de episodios de diarrea, mejoría de letargo y disminución de la temperatura. La paciente no inicia trabajo de parto durante la atención, por lo que se decide continuar controles ambulatorios estrictos por endocrinología y ginecología de alto riesgo, quienes programan parto a las 40 semanas y se decide terapia con yodo posterior a periodo de lactancia.

\section{Discusión}

La tormenta tiroidea es una complicación poco frecuente de un trastorno muy común como lo es la tirotoxicosis, la cual puede ser desencadenada por estrés severo y está asociada a una elevada morbimortalidad, con falla multiorgánica potencialmente mortal ${ }^{(2,4)}$.

La prevalencia de la enfermedad se ha reportado en $1 \%$ en los pacientes hospitalizados por hipertiroidismo, con una incidencia de 0,2/100.000 personas-año representando el $0,22 \%$ de todos los pacientes con tirotoxicosis y el 5,4\% de los pacientes con tirotoxicosis hospitalizados. Se presenta con mayor frecuencia en las mujeres que en los hombres con una relación 3:1. El pronóstico es desfavorable con una mortalidad del 10\%-30\% por falla multiorgánica y falla cardiaca principalmente, la cual se reduce con una terapia adecuada si se instaura rápidamente $\mathrm{e}^{(2,3,5)}$.

En Colombia no hay estudios para determinar la frecuencia de esta entidad que, aunque es una complicación rara, es muy importante tenerla en cuenta para dar manejo oportuno a estos pacientes y evitar complicaciones con desenlaces fatales.

En la mayoría de los casos, la tormenta tiroidea se produce después de un evento desencadenante. El hipertiroidismo sin tratamiento o con mal control junto con otros factores puede llegar a precipitar una tormenta tiroidea ${ }^{(2,6,7)}$. Los factores desencadenantes se enumeran en la tabla 1.

El cuadro clínico característico de tormenta tiroidea no está relacionado con los niveles de hormonas tiroideas. Los pacientes con tormenta tiroidea tienen una mayor cantidad de sitios de unión de catecolaminas que los sujetos con hipertiroidismo que no la desarrollan, al parecer existe una mayor disponibilidad de los receptores adrenérgicos y una reducción del enlace de hormona tiroidea a la globulina de unión a la hormona tiroidea, de tal suerte que la filtración de catecolaminas provocada por un evento agudo o factor desencadenante precipita tormenta tiroidea ${ }^{(3)}$.

La tormenta tiroidea se desencadena cuando la capacidad de los mecanismos de compensación termorreguladores, car-
Tabla 1. Factores precipitantes para tormenta tiroidea

- Uso irregular o la interrupción de los fármacos antitiroideos

- Infección

- Enfermedad cerebrovascular

- Cetoacidosis diabética

- Ejercicio intenso

- Estrés emocional severo

- Cardiopatía isquémica

- Trauma

- Insuficiencia adrenocortical

- Cirugía no tiroidea

- Administración de medio de contraste yodado

- La terapia con yodo radioactivo

- Hipertiroidismo facticio

- Asociado a interferón o amiodarona

- La extracción de los dientes

- Otros

diovasculares y metabólicos del paciente son superados por el exceso de hormona tiroidea para mantener una situación de equilibrio. Esto puede aparecer con mayor frecuencia en hipertiroidismos de cualquier etiología, entre ellos: la enfermedad de Graves-Basedow, el adenoma tóxico y el bocio multinodular tóxico y con menor frecuencia en carcinomas tiroideos secretores, adenomas hipofisiarios productores de TSH y con el estroma ovárico, además en hipertiroidismo facticio, medicamentos como amiodarona, interferón y tras administración exógena de yodo ${ }^{(8)}$.

El diagnóstico clínico es fundamental, ya que los exámenes de laboratorio no muestran diferencias notorias con el resto de pacientes con hipertiroidismo. La presentación clínica consiste en fiebre, taquicardia sinusal con o sin falla cardiaca, incluso fibrilación auricular, síntomas gastrointestinales como náusea, vómito, diarrea y síntomas neurológicos que varían desde confusión hasta coma. Se encuentra TSH suprimida con T4 y T3 elevados ${ }^{(9)}$.

Los síntomas de la tormenta tiroidea no son diferentes de un hipertiroidismo no controlado; sin embargo, en un estudio de cohorte Angell T. et al. en EE.UU., 2015, compararon el diagnóstico, las diferencias en la clasificación y otras características clínicas de los pacientes hospitalizados con hipertiroidismo y tormenta tiroidea, y encontraron que la alteración del estado mental fue la única característica clínica significativamente diferente entre las dos entidades y que estos pacientes tenían peores desenlaces en cuanto a mortalidad, mayor estancia hospitalaria y en UCI, además de mayores requerimien- 
tos de ventilación mecánica, de tal manera que los pacientes con disfunción del sistema nervioso central se benefician de tratamientos más agresivos ${ }^{(10)}$.

Los criterios más utilizados para el diagnóstico de tormenta tiroidea son los propuestos por Burch and Wartofsky en 1993 e incluyen: disfunción termorreguladora, compromiso del sistema nervioso central que involucra agitación, delirio, psicosis, estupor y coma, compromiso gastrointestinal, disfunción cardiovascular: taquicardia, arritmias, insuficiencia cardiaca congestiva y factor desencadenante ${ }^{(7,9,11)}$. Los criterios de Burch and Wartofsky, 1993 se encuentran en la tabla 2. Es importante tener en cuenta el diagnóstico clínico, ya que muchos de los pacientes que ingresan a un servicio de urgencias, por cualquiera de las alteraciones mencionadas, no se estudian debidamente, aumentando la morbilidad y mortalidad. En los dos casos presentados, las pacientes tenían puntuaciones en la escala diagnóstica por encima de 45 , lo cual confirma la tormenta tiroidea, de manera que se instauró un tratamiento rápido y oportuno con adecuados resultados en ambas pacientes.

Los principios del tratamiento van encaminados a reducir el exceso de hormona tiroidea en la sangre y sus efectos nocivos a nivel periférico y se basan en la experiencia clínica y estudios de casos, ya que no existen estudios prospectivos.

El tratamiento inicial es la reanimación. Se debe iniciar oxígeno de bajo o alto flujo e incluso ventilación mecánica en caso de hipoxemia severa, además, infusión de cristaloides y corrección hidroelectrolítica tan pronto como sea posible, ya que el estado de hipermetabolismo puede llevar a hipertermia con deshidratación e incluso inestabilidad hemodinámica. Se pueden iniciar antipiréticos con medios físicos pero los salicilatos (por ejemplo aspirina) deben ser evitados, ya que compiten con las hormonas tiroideas por sus transportadores ${ }^{(8)}$. Es muy importante la terapia de apoyo y el tratamiento de los factores precipitantes.

Los bloqueadores beta: propranolol 1 - 2 mg intravenoso o 40 a 80 mg por vía oral cada cuatro a seis horas, ajustado según la frecuencia cardiaca y la tensión arterial, bloquea la unión de las catecolaminas a los receptores betaadrenérgicos y, por otro lado, reduce la conversión periférica de $\mathrm{T} 4$ a T3 ${ }^{(11,12)}$.

El esmolol, otra alternativa de tratamiento, es un betabloqueador cardioselectivo de acción corta. Se utiliza dosis de carga de 250 a $500 \mathrm{mcg} / \mathrm{kg}$, seguida de infusión de 50 a 100 $\mathrm{mcg} / \mathrm{kg}$ por minuto, permitiendo una rápida acción y reducción de efectos adversos sobre todo en pacientes con asma y EPOC $^{(11,13)}$.

Para el bloqueo de la síntesis de hormona tiroidea se utilizan las tionamidas con propiltiouracilo a dosis de carga 500 a $1.000 \mathrm{mg}$ y continuar a $250 \mathrm{mg}$ cada 4 horas, este fármaco no sólo bloquea la síntesis de la hormona sino que también bloquea la conversión de T4 a T3. Otro fármaco utilizado es el metimazol en dosis de 60 a $80 \mathrm{mg}$ al día ${ }^{(11)}$. Estos medicamentos también se pueden administrar en forma de supositorio
Tabla 2. Criterios diagnósticos para tormenta tiroidea

\begin{tabular}{|l|c}
\hline Parámetro diagnóstico & Puntos \\
\hline Disfunción termorreguladora & 5 \\
\hline Temperatura & 10 \\
\hline $37,2-37,7$ & 15 \\
$37,8-38,3$ & 20 \\
$38,4-38,8$ & 25 \\
$38,9-39,3$ & 30 \\
$39,4-39,9$ & \\
$>39,9$ &
\end{tabular}

Compromiso del sistema nervioso central

Ausente

Leve: agitación

0

Moderado: delirium, psicosis, letargia extrema

Severo: convulsiones, coma

5

15

20

25

30

Compromiso gastrointestinal

Ausente

Moderado: diarrea, náuseas, vómito, dolor abdominal 10

Severo: ictericia

Disfunción cardiovascular

Taquicardia

90-109

110-119

120-129

10

30-139

15

130-139

20

$>140$

25

Fibrilación auricular

Ausente

Presente

0

10

Falla cardiaca congestiva

\begin{tabular}{l|l}
\hline Ausente & 0 \\
Leve & 5 \\
Moderada & 10 \\
Severa & 20 \\
\hline Evento precipitante & 0 \\
\hline Ausente & 10 \\
Presente &
\end{tabular}

$>$ 45: Tormenta tiroidea

25 - 44: Sugestivo de tormenta tiroidea

$<25$ : Tormenta tiroidea poco probable

Adaptado de Burch and Wartofsky, 1993 
o enema con preparaciones especiales o preparaciones para administración intravenosa en casos seleccionados.

Las soluciones de yodo bloquean la síntesis de hormona y bloquean su liberación, éstas deben ser administradas una hora después de tomar la primera dosis de tionamida con el fin de reducir los niveles de hormona tiroidea causada por la ingesta de gran cantidad de yodo, fenómeno de Wolff - Chaikoff. El más utilizado es yoduro de potasio (Lugol) 5 gotas $(0,25 \mathrm{ml}$ o $250 \mathrm{mg}$ ) vía oral cada 6 horas, aunque existen otras alternativas, como la presentación endovenosa de yoduro de sodio 500 - 1000 mg al día e intrarrectal. Esto puede ser útil durante las primeras 72 horas, ya que posteriormente puede presentarse un fenómeno de escape o fenómeno de Jod-Basedow en donde ocurre síntesis y liberación de hormonas tiroideas tras su administración, que agravaría el hipertiroidismo ${ }^{(3,4,8,11)}$. El ácido Iopanoico es un agente de contraste yodado que podría llegar a ser útil en el manejo, con una dosis de $1 \mathrm{~g}$ endovenoso cada 8 horas durante las primeras 24 horas y continuar a 500 mg dos veces al día ${ }^{(12)}$.

Como alternativa al yodo inorgánico se puede utilizar carbonato de litio $300 \mathrm{mg}$ cada 6 u 8 horas, el cual inhibe la proteólisis de coloide, limitando la liberación de hormona tiroidea preformada al torrente sanguíneo ${ }^{(3)}$.

Los glucocorticoides son utilizados para bloquear la conversión de T4 a T3 y como profilaxis para la insuficiencia adrenal relativa que coexiste con la tirotoxicosis. El más utilizado es la hidrocortisona dosis de carga $300 \mathrm{mg}$ intravenosa y continuar a $100 \mathrm{mg}$ cada 8 horas y como medicamento alternativo la dexametasona $2 \mathrm{mg}$ IV cada 6 horas $^{(8,11,12)}$.

La colestiramina $4 \mathrm{~g}$ vía oral cada 6 a 12 horas puede ser útil debido a la reducción de la reabsorción de la hormona tiroidea metabolizada desde la circulación enterohepática ${ }^{(12)}$

La tiroidectomía se realiza en aquellos pacientes con disfunción de órganos, refractaria al tratamiento o cuando se presenta toxicidad con las tionamidas; sin embargo, los criterios para decidir la cirugía y el momento en el cual se debe realizar no se han estudiado con detalle. A pesar de esto, se han encontrado tasas de mortalidad global para pacientes sometidos a tiroidectomía en tormenta tiroidea con enfermedad de Graves del $10 \%$, asociadas con un riesgo de exacerbación de la tormenta si los niveles de hormonas tiroideas preoperatoria son altos $^{(14)}$.

El yodo radiactivo no es un tratamiento recomendado en tormenta tiroidea, ya que puede agravar la situación con exacerbación de los síntomas e incluso puede ser un desencadenante de tormenta tiroidea, esto se debe a una tiroiditis por radiación con destrucción de folículos tiroideos y liberación de cantidades elevadas de hormonas tiroideas y tiroglobulina a la circulación $^{(15,16)}$.
Otras terapias alternativas como la plasmaféresis se han utilizado cuando el tratamiento tradicional no ha tenido éxito y como preparación para tiroidectomía. La plasmaféresis elimina citocinas, anticuerpos y hormonas tiroideas del plasma. Es el método más rápido para mejorar la condición clínica del paciente y puede ser empleado cuando hay síntomas neurológicos o cardiovasculares que amenacen la vida sin esperar el efecto de los medicamentos antitiroideos ${ }^{(17)}$.

El segundo caso presentado se trata de una mujer en estado de embarazo a quien se le inicia manejo para tormenta tiroidea con adecuada respuesta clínica y sin complicaciones obstétricas. La tormenta tiroidea se presenta con baja frecuencia, constituyendo un riesgo para la madre y el feto con efectos deletéreos como: hipertensión, falla cardiaca y preeclampsia en mujeres embarazadas, además abortos, parto pretérmino, desprendimiento de la placenta y efectos en el feto como: taquicardia fetal, cardiomegalia, hidropesía, edad ósea avanzada, restricción del crecimiento intrauterino y mortinato. No se ha asociado el embarazo como factor desencadenante de tormenta tiroidea, por lo que es necesario buscar factores precipitantes. Se debe iniciar terapia con antitiroideos de los cuales se recomienda en el primer trimestre propiltiouracilo, ya que su uso no está asociado con anomalías congénitas o metimazol en el caso de la alergia a propiltiouracilo. En el segundo y tercer trimestre se prefiere el uso de metimazol debido a la posible hepatotoxicidad que se puede presentar con propiltiouracilo.

La cirugía está indicada en el segundo trimestre en el caso de reacciones adversas severas a los antitiroideos, cuando no hay respuesta al manejo. La terapia con yodo radiactivo está prohibida en el embarazo ${ }^{(18)}$. Las mujeres que requieren tratamiento antitiroideo después del parto pueden continuar, incluso durante la lactancia, con las dosis más bajas toleradas (hasta $30 \mathrm{mg}$ metimazol o $150 \mathrm{mg}$ propiltiouracilo) ${ }^{(19,20)}$.

\section{Conclusión}

La tormenta tiroidea es una complicación rara de una enfermedad relativamente frecuente como es el hipertiroidismo, con altas tasas de mortalidad y cuyo diagnóstico se basa en el juicio clínico, por lo que se deben aplicar los criterios diagnósticos para hacer un tratamiento oportuno basado en el bloqueo inmediato de la síntesis de la hormona, evitar la liberación y tratamiento de los efectos periféricos, así como la búsqueda y tratamiento de los factores desencadenantes, de manera que se pueda llegar a reducir los desenlaces mortales y disminuir la estancia hospitalaria en piso y en UCI. En Colombia faltan estudios para determinar la verdadera frecuencia de la enfermedad que, aunque es rara, debe ser tenida en cuenta en los servicios de urgencias para evitar efectos deletéreos en los pacientes. 


\section{Referencias}

1. Parasa M, Chinthakunta BK, Vemuri NN, Shaik MS. Out of the blue! Thyroid crisis. Anesthesia, essays and researches. 2015;9(1):130-2.

2. Akamizu T, Satoh T, Isozaki O, Suzuki A, Wakino S, Iburi T, et al. Diagnostic criteria, clinical features, and incidence of thyroid storm based on nationwide surveys. Thyroid : official journal of the American Thyroid Association. 2012;22(7):661-79.

3. Papi G, Corsello SM, Pontecorvi A. Clinical concepts on thyroid emergencies. Frontiers in endocrinology. 2014;5:102.

4. Rojas G W. Urgencias en tiroides: tormenta tiroidea y coma mixedematoso. En: Ardila A E, Barbosa M J, Dorado P L, et al, editores. Fascículo alteraciones endocrinas en urgencias. Bogotá: Producciones Científicas Ltda; 2012. p. $53-62$.

5. Almanzar A., Mora L, Valderrama L, Barón G. Serie de casos: tirotoxicosis y tormenta tiroidea. Dovela científica (internet). 2011 (citado 09 Jul 2015); 1 (4): 1-8. Disponible en: http://www.hospitaloccidentekennedy.gov.co/ archivos/File/Dovela_Vol_1_No_/Casos_clinicos.html.

6. Hampton J. Thyroid gland disorder emergencies: thyroid storm and myxedema coma. AACN advanced critical care. 2013;24(3):325-32.

7. Burch HB, Wartofsky L. Life-threatening thyrotoxicosis. Thyroid storm. Endocrinology and metabolism clinics of North America. 1993;22(2):263-77.

8. Rámiz M., Gutiérrez A., De la Guerra C. et al. Crisis tirotóxica (tormenta tiroidea). A propósito de dos observaciones. Gac Med Bilbao. 2010;107(2):6467.

9. Klubo-Gwiezdzinska J, Wartofsky L. Thyroid emergencies. The Medical clinics of North America. 2012;96(2):385-403.

10. Angell TE, Lechner MG, Nguyen CT, Salvato VL, Nicoloff JT, LoPresti JS. Clinical features and hospital outcomes in thyroid storm: a retrospective cohort study. The Journal of clinical endocrinology and metabolism. 2015;100(2):451-9.

11. Bahn Chair RS, Burch HB, Cooper DS, Garber JR, Greenlee MC, Klein I, et al Hyperthyroidism and other causes of thyrotoxicosis: management guidelines of the American Thyroid Association and American Association of Clinical Endocrinologists. Thyroid : official journal of the American Thyroid Association. 2011;21(6):593-646.

12. Carroll R, Matfin G. Endocrine and metabolic emergencies: thyroid storm Therapeutic advances in endocrinology and metabolism. 2010;1(3):139-45.

13. Duggal J, Singh S, Kuchinic P, Butler P, Arora R. Utility of esmolol in thyroid crisis. The Canadian journal of clinical pharmacology = Journal canadien de pharmacologie clinique. 2006;13(3):e292-5.

14. Uchida $\mathrm{N}$, Suda $\mathrm{T}$, Ishiguro $\mathrm{K}$. Thyroidectomy in a patient with thyroid storm: report of a case. Surgery today. 2015;45(1):110-4.

15. Aulet RM, Wein RO, Siegel RD. Surgical management of an atypical presentation of a thyroid storm. International journal of endocrinology and metabolism. 2014;12(2):e13539.

16. Ahumada J.J. Terapia del hipertiroidismo. Acta Medica Colombiana Vol. 13 No. 1 (Enero-Febrero) 1988.

17. Muller C, Perrin P, Faller B, Richter S, Chantrel F. Role of plasma exchange in the thyroid storm. Therapeutic apheresis and dialysis : official peer-reviewed journal of the International Society for Apheresis, the Japanese Society for Apheresis, the Japanese Society for Dialysis Therapy. 2011;15(6):522-31.

18. Yoshihara A, Noh J, Yamaguchi T, Ohye H, Sato S, Sekiya K, et al. Treatment of graves' disease with antithyroid drugs in the first trimester of pregnancy and the prevalence of congenital malformation. The Journal of clinical endocrinology and metabolism. 2012;97(7):2396-403.

19. Glinoer D. The systematic screening and management of hypothyroidism and hyperthyroidism during pregnancy. Trends in endocrinology and metabolism: TEM. 1998;9(10):403-11.

20. Restrepo O. Hipertiroidismo durante el embarazo: enfoque y manejo. Revista Colombiana de Obstetricia y Ginecología Vol. 52 No. 3 - 2001. 